\title{
Structure-Function Relationships of Healthy and Osteoarthritic Human Tibial Cartilage: Experimental and Numerical Investigation
}

\author{
Mohammadhossein Ebrahimi (i), ${ }^{1,2}$ Mikael J. Turunen, ${ }^{1,3}$ \\ Mikko A. Finnilä, ${ }^{2}$ Antti Joukainen, ${ }^{4}$ Heikki Kröger, 4 \\ Simo SaArakkala, ${ }^{2,5}$ Rami K. Korhonen, ${ }^{1}$ and Petri Tanska id ${ }^{1}$ \\ ${ }^{1}$ Department of Applied Physics, University of Eastern Finland, POB 1627, 70211 Kuopio, Finland; ${ }^{2}$ Research Unit of Medical \\ Imaging, Physics and Technology, Faculty of Medicine, University of Oulu, Oulu, Finland; ${ }^{3}$ SIBlabs, University of Eastern \\ Finland, Kuopio, Finland; ${ }^{4}$ Kuopio University Hospital, Kuopio, Finland; and ${ }^{5}$ Department of Diagnostic Radiology, Oulu \\ University Hospital, Oulu, Finland
}

(Received 17 January 2020; accepted 26 June 2020; published online 9 July 2020)

Associate Editor Eric M. Darling oversaw the review of this article.

\begin{abstract}
Relationships between composition, structure and constituent-specific functional properties of human articular cartilage at different stages of osteoarthritis (OA) are poorly known. We established these relationships by comparison of elastic, viscoelastic and fibril-reinforced poroelastic mechanical properties with microscopic and spectroscopic analysis of structure and composition of healthy and osteoarthritic human tibial cartilage $(n=27)$. At a low frequency $(0.005 \mathrm{~Hz})$, proteoglycan content correlated negatively and collagen content correlated positively with the phase difference (i.e. tissue viscosity). At a high-frequency regime $(>0.05 \mathrm{~Hz})$, proteoglycan content correlated negatively and collagen orientation angle correlated positively with the phase difference. Proteoglycans were lost in the early and advanced OA groups compared to the healthy group, while the superficial collagen orientation angle was greater only in the advanced OA group compared to the healthy group. Simultaneously, the initial fibril network modulus (fibril pretension) was smaller in the early and advanced OA groups compared to the healthy group. These findings suggest different mechanisms contribute to cartilage viscosity in low and high frequencies, and that the loss of superficial collagen pretension during early $\mathrm{OA}$ is due to lower tissue swelling (PG loss), while in advanced OA, both collagen disorganization and lower swelling modulate the collagen fibril pretension.
\end{abstract}

Keywords-Proteoglycan, Collagen fibril network, Fibril-reinforced poroelastic, Fourier transform infrared spectroscopy, Mechanical properties, Polarized light microscopy, Digital densitometry.

Address correspondence to Mohammadhossein Ebrahimi, Department of Applied Physics, University of Eastern Finland, POB 1627, 70211 Kuopio, Finland. Electronic mail: mohammadhossein. ebrahimi@uef.fi and ebrahimi.bioengineering@gmail.com

\section{INTRODUCTION}

Articular cartilage provides the joint with smooth movements during locomotion. With its biphasic nature, cartilage is highly resilient and dissipative tissue. The fluid phase makes up approximately $70-80 \%$ of the wet weight of the tissue. The solid phase consists mainly of proteoglycans (PGs) $(5-7 \%$ of the wet weight) and collagen fibril (mainly type II, $15-23 \%$ of the wet weight). ${ }^{29,35-37}$ The content, structure, and interactions between these main constituents control the mechanical behavior of cartilage. Cartilage responses to impacts and high-strain loads are mainly regulated by the collagen fibrils network and high interstitial fluid pressure. Under prolonged loading, fluid flows out of the tissue and PGs mainly control the equilibrium stiffness of the tissue. ${ }^{37,51}$

Osteoarthritis (OA) is a degenerative joint disease causing disabilities to large populations worldwide (e.g. approximately $9 \%$ of Americans ${ }^{26}$ ) and thus accounts for billions of dollars in the healthcare system. During the OA progression, tissue constituents experience substantial changes, such as loss of PGs and collagen, disorganization of collagen fibril network, and increased interstitial fluid content. ${ }^{14,25,49}$ These changes affect significantly the load-bearing capacity of the tissue.

Recent studies have employed animal models, such as steer, ${ }^{47}$ pony ${ }^{50}$ and most recently fetal ovine cartilage $^{6}$ to investigate the relationships between structure, composition, and function of cartilage in OA. Animal 
models are very convenient to investigate complex biological processes promptly in well-controlled study cohorts (e.g. controlling age and gender). The onset and progression of different subtypes of OA, such as post-traumatic OA, can also be investigated in small $^{10,21,41}$ and large animal models. ${ }^{1,2}$ Compared to small animal models, the use of large animal models enables studying the mechanisms of OA in a wider time frame, approaching the slow OA development in humans. Yet, understanding how native human tissue alters in OA provides novel insights into the disease, which is not possible to obtain directly from animal models. Investigations of human tissues can also elucidate how the animal model-based knowledge is transferrable to humans.

Natural differences between animal and human tissues have been reported in the literature. ${ }^{3,9}$ Structurefunction relationships have been studied in human hip joint cartilage ${ }^{31}$ and human patellar cartilage. ${ }^{23}$ However, structure-function relationships in human tibial and femoral cartilage are still not well-known. This poses a challenge, for example, in a computational modeling of the knee joint. A better knowledge of structure-function relationships of cartilage will improve the accuracy of the models. In addition, structure-function relationships are important for in vitro studies to understand cell-tissue interactions. ${ }^{8}$ Therefore, a comprehensive investigation of the relationships between structure, composition and function of human tibial cartilage is essential.

The swelling pressure is known to be an important contributor to the mechanical behavior of cartilage. This has been investigated specifically through fibrilreinforced poroviscoelastic modeling of articular cartilage. ${ }^{22,28,45}$ The swelling pressure caused by the PG matrix modulates the collagen fibril reinforcement inside the cartilage. ${ }^{55}$ During transient conditions, collagen fibrils of the superficial zone, as well as fluid pressurization, carry a considerable amount of load. The loss of swelling and fluid pressurization may affect superficial collagen fibrils by diminishing their pretension, thus, impairing the contribution of collagen to the mechanical function of the tissue. ${ }^{30,45}$ However, none of the studies correlated the model-derived mechanical properties of articular cartilage with the structural and composition properties. The fibril-reinforced poroelastic (FRPE) material model incorporates the main constituents of cartilage and it can differentiate their contribution to the mechanical response. Combined with sample-specific finite element modeling, the FRPE material has shown the capability to accurately simulate experimentally derived data in dynamic and stress-relaxation tests. ${ }^{24}$ Fibril-reinforcement in the model, emphasized by high fluid pressurization, accounts for cartilage behavior particularly in short-term loading, ${ }^{27}$ while the non-fibrillar matrix is mainly responsible for the equilibrium response.

In our recent study, ${ }^{15}$ we observed that the severity of OA in human tibial cartilage was strongly associated with changes in the constituent-specific FRPE mechanical properties as well as elastic and viscoelastic properties. Comparing to the healthy tissue, we observed a great reduction in the initial fibril network modulus (related to the pretension of the collagen fibrils) and the non-fibrillar matrix modulus (related to the PG content). Moreover, elastic and viscoelastic mechanical properties exhibited negative and positive correlations, respectively, with the severity of OA.

In the present study, our objective was to investigate whether these previously observed alterations in the elastic, viscoelastic and constituent-specific FRPE mechanical properties at different stages of OA could be explained by changes in the structure and composition of cartilage. We hypothesized that:

(1) the loss of initial fibril network modulus at different stages of OA is because of loss of PGs (loss of swelling pressure) and loss of collagen content

(2) the PG content is the main contributor to the viscous response of cartilage (i.e. phase difference between sinusoidal displacement and force)

This study provides novel knowledge on structurefunction relationships of human tibial cartilage at different stages of OA. This knowledge is also important to improve knee joint and multiscale modeling.

\section{MATERIALS AND METHODS}

\author{
Sample Preparation, Mechanical Testing \\ and Determination of the Elastic, Viscoelastic \\ and Constituent Specific Material Parameters
}

As the biomechanical properties were reported in our previous study, ${ }^{15}$ a brief recap of sample preparation, mechanical testing and determination of the elastic, viscoelastic and constituent-specific (FRPE) parameters is provided in the supplementary material.

The process and use of the human tissue were approved by the National Authority for Medicolegal Affairs and the ethical committee of North Savo Hospital District (Ethical Permission Number 134/ 13.02.00/2015).

In the current study, human osteochondral samples $(n=27)$ from tibial cartilage of 7 cadavers were processed to determine quantitative compositional and structural properties (Fig. 1). The OA severity of the samples was defined using the Osteoarthritis Research Society International (OARSI) histopathological 


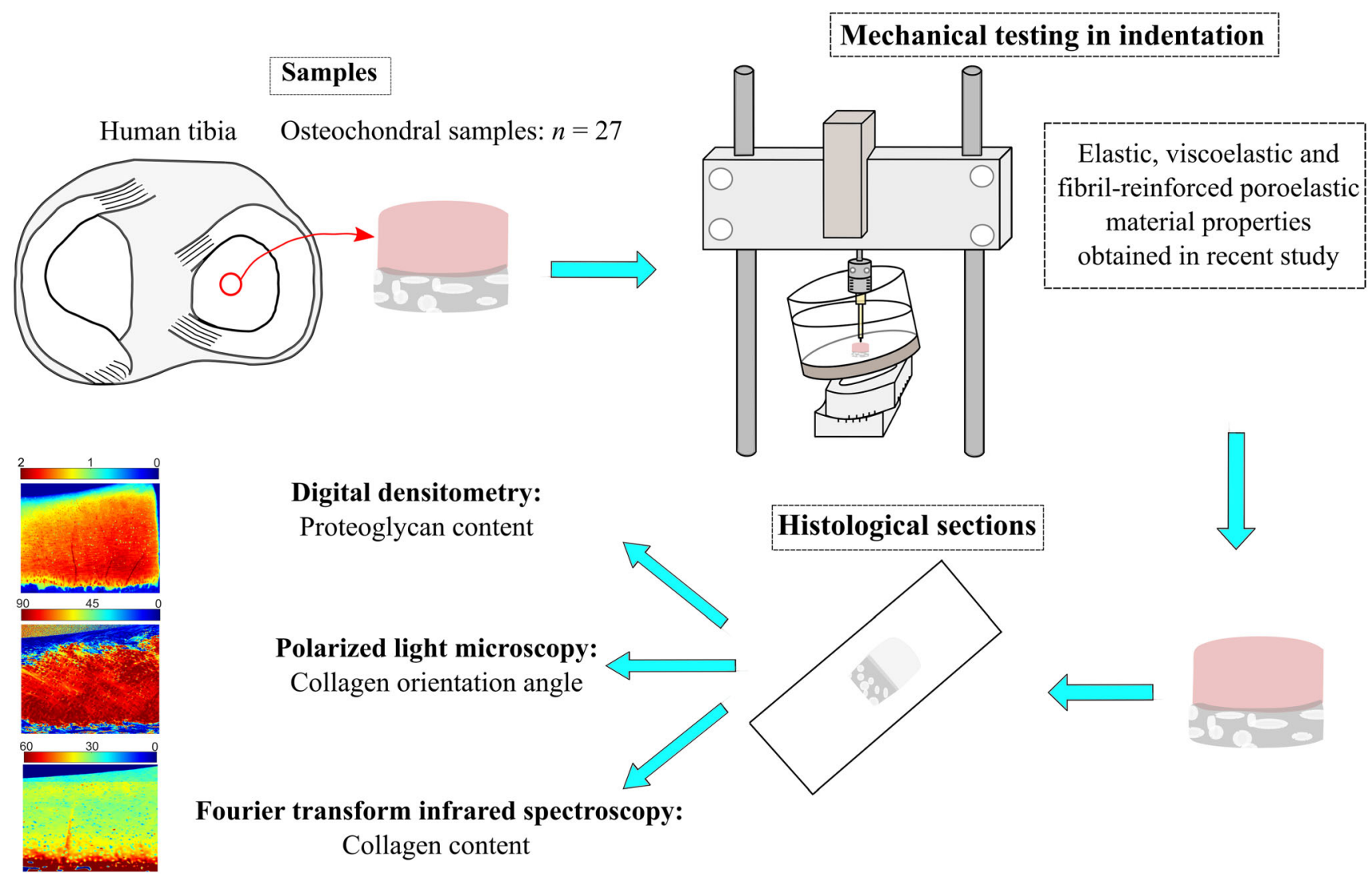

FIGURE 1. Workflow of the study. First, biomechanical measurements were conducted for the osteochondral samples obtained from tibia. Then, the samples were formalin-fixed, decalcified and embedded in paraffin tissue blocks. The blocks were sectioned and stained for OARSI grading. These steps were conducted in our previous study. ${ }^{15}$ In this study, the blocks underwent additional quantitative histological analyses including digital densitometry, polarized light microscopy and Fourier transform infrared spectroscopy.

grading system from Safranin-O sections. Samples were then grouped to healthy (OARSI grades $0-1$ ), early OA (OARSI grades 2-3) and advanced OA (OARSI grade 4).

\section{Microscopical and Spectroscopical Analyses}

In the current study, the samples were processed to determine quantitative compositional and structural properties using digital densitometry (DD), polarized light microscopy (PLM) and Fourier transform infrared spectroscopy (FTIR). Following the indentation tests, the samples were fixed in formalin and decalcified. Then, the samples were dehydrated in graded ethanol solutions and embedded in paraffin. ${ }^{32,41} \mathrm{Sec}-$ tions were prepared from the middle of the sample and perpendicular to the surface providing sections from the same area in which the biomechanical indentation test was performed. For DD measurements, $3 \mu \mathrm{m}$ thick sections were stained with Safranin-O. However, for PLM and FTIR measurements, $5 \mu$ m thick sections were prepared. After cutting the sections, the paraffin was removed. The total number of sections for each set of microscopy/spectroscopy measurements was 81 (i.e. 3 adjacent sections per sample).

Digital densitometry was performed using a conventional light microscope (Nikon Microphot FXA, Nikon Co., Tokyo, Japan), CCD-camera (Hamamatsu ORCA-ER, Hamamatsu Photonics, Hamamatsu, Japan) and monochromator $(\lambda=492 \pm 5 \mathrm{~nm})$ to capture grayscale images of Safranin-O stained sections (pixel size $=3.09 \times 3.09 \mu \mathrm{m}$ ). The system was calibrated with filters (optical density values $0.0,0.15,0.3$, $0.6,1.0,1.3,1.6,2.0,2.6$ and 3.0) (Schott, Mainz, Germany). ${ }^{12,41}$ Thus, optical density which is a quantitative estimator of the PG content of the samples was acquired. Three slices per sample were measured. Based on our analyses, the use of three sections provided consistent results within a sample (coefficient of variation $<0.1$, see supplementary material). Three sections have also been used in several previous studies. ${ }^{48,52,54}$

A conventional light microscope (Nikon Diaphot TMD, Nikon, Inc., Shinagawa, Tokyo, Japan, pixel 
size $=2.53 \times 2.53 \mu \mathrm{m})$ equipped with Abrio PLM system (CRi, Inc., Woburn, MA, USA) was used to determine the orientation angle of the collagen fibrils. ${ }^{19,46}$ The Abrio system uses a circular polarizer consisting of two liquid crystal polarizers to assess the main orientation of the collagen fibrils in each pixel. Three sections per sample were measured. ${ }^{48,52,54}$

Agilent Cary 600 spectrometer coupled with Cary 610 FTIR microscope (Agilent Technologies, Santa Clara, CA, USA) was used to obtain spectral data for the analysis of the collagen content. Infrared light absorption spectrum with wavenumber ranging from 3800 to $750 \mathrm{~cm}^{-1}$ was collected pixel-by-pixel. To increase the signal to noise ratio, 8 scans per pixel were acquired. The spatial pixel size was $5.5 \times 5.5 \mu \mathrm{m}$ and the spectral resolution was set to $4 \mathrm{~cm}^{-1}$. Following data acquisition, a constant baseline correction for the spectrum ranging from 2000 to $900 \mathrm{~cm}^{-1}$ was conducted. The Amide I region $\left(1720-1595 \mathrm{~cm}^{-1}\right)$ of the infrared spectrum was analyzed and used to estimate the collagen content. ${ }^{48,49}$ Three sections per sample were measured. $^{48,52,54}$

The compositional and structural analyses were conducted in a $1.5 \mathrm{~mm}$ wide region of interest (averaging the pixel values in a horizontal direction) across the whole tissue depth using a custom-made code in Matlab software (v7.10.0, Mathworks Inc., MA, USA). Then, the three profiles obtained from three adjacent sections of each sample were averaged. Therefore, the depth-wise variations in the PG content, collagen content, and collagen orientation angle could be investigated in relation to the biomechanical and constituent-specific properties of cartilage.

\section{Statistical Analyses}

To test our hypotheses, the measured structure and composition of human tibial cartilage were compared with the elastic, viscoelastic and constituent-specific FRPE material properties. The mechanical material properties were adopted from our previous study. ${ }^{15}$

The depth-wise changes in the cartilage structure and composition were compared between different $\mathrm{OA}$ groups using a linear mixed-effects model. Statistical power analyses were conducted to elucidate the power of statistical models and post hoc tests (see supplementary material).

Furthermore, a linear multivariable regression analysis was conducted to evaluate structure-function relationships. Analyses were conducted using IBM SPSS Statistics (version 25, IBM Corporation, Armonk, NY, USA; see supplementary materials).

\section{RESULTS}

Representative PG content, collagen content, and collagen orientation angle images from different $\mathrm{OA}$ groups are shown in Fig. 2.

\section{Depth-Wise Structural and Compositional Alterations of Human Tibial Cartilage at Different Stages of OA}

The depth-wise profiles of the composition/structure and point-by-point comparisons between the OA group profiles are shown in Fig. 3. Consistent with the changes in the equilibrium and non-fibrillar matrix modulus observed in our previous study, ${ }^{15}$ the PG loss occurred from 0 to $12 \%$ of the cartilage thickness in the early $O A$ group compared to the healthy group. However, the PG loss in the advanced $O A$ group compared to the healthy group progressed further, up to $37 \%$ of the cartilage thickness.

The superficial collagen fibrils were less parallel to the surface (i.e. greater orientation angle) in the early $O A$ group compared to the healthy group in the superficial cartilage (from 0 to $4 \%$ of the thickness). In the advanced $O A$ group, the collagen fibrils were less parallel to the surface up to $12 \%$ of the cartilage thickness compared to the healthy group. Moreover, the collagen orientation angle was greater in the $a d-$ vanced $O A$ group compared to the early $O A$ group at the depths ranging from 10 to $18 \%$ of the cartilage thickness.

The collagen content was not different between the early $O A$ and healthy groups, while it was smaller in the advanced $O A$ compared to the healthy group at the depths ranging from 17 to $52 \%$ of the cartilage thickness. Interestingly, the collagen content was also smaller at the depths ranging from 31 to $43 \%$ when the advanced $O A$ group was compared to the early $O A$ group.

\section{Structure-Composition Correlation Analysis with the Elastic and Viscoelastic Mechanical Parameters}

The PG content showed positive linear correlations with the equilibrium and initial instantaneous moduli at all tissue depths (Table 1, Supplementary Tables S1 and S2). The PG content also exhibited a positive linear correlation with the dynamic modulus at all depths, except at the depths of $0-5 \%$. Importantly, the PG content analyzed up to $20 \%$ of the tissue thickness exhibited a negative linear correlation with the phase difference measured with $0.005 \mathrm{~Hz}$ sinusoidal loading frequency. However, the PG content of bulk cartilage exhibited a negative correlation with the phase differ- 
Fourier transform infrared microspectroscopy

Collagen content (Amide I)
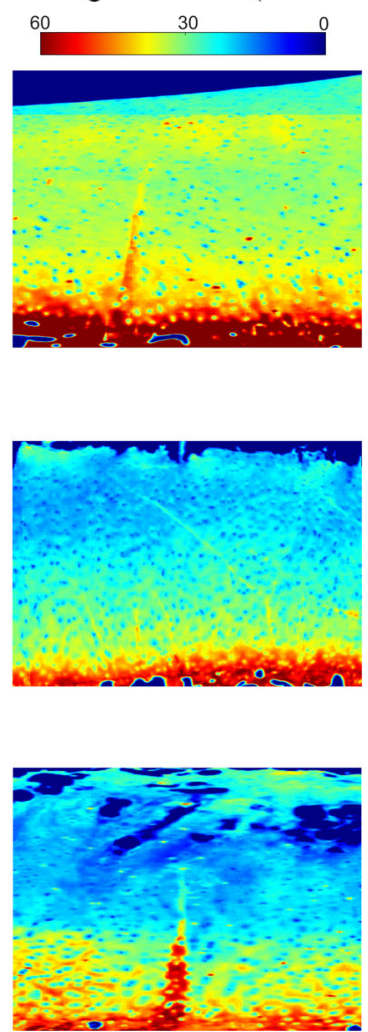

Polarized light microscopy Collagen orientation angle $\left(0^{\circ}=\right.$ parallel to surface $)$
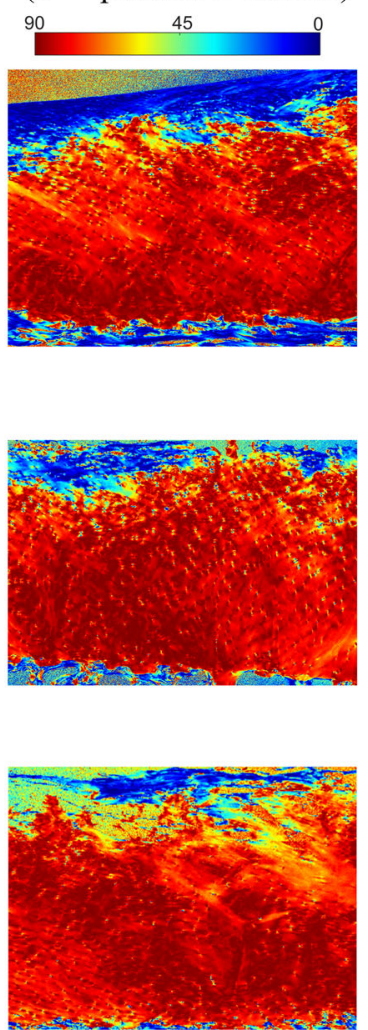

Digital densitometry

Proteoglycan content (optical density, absorbance)
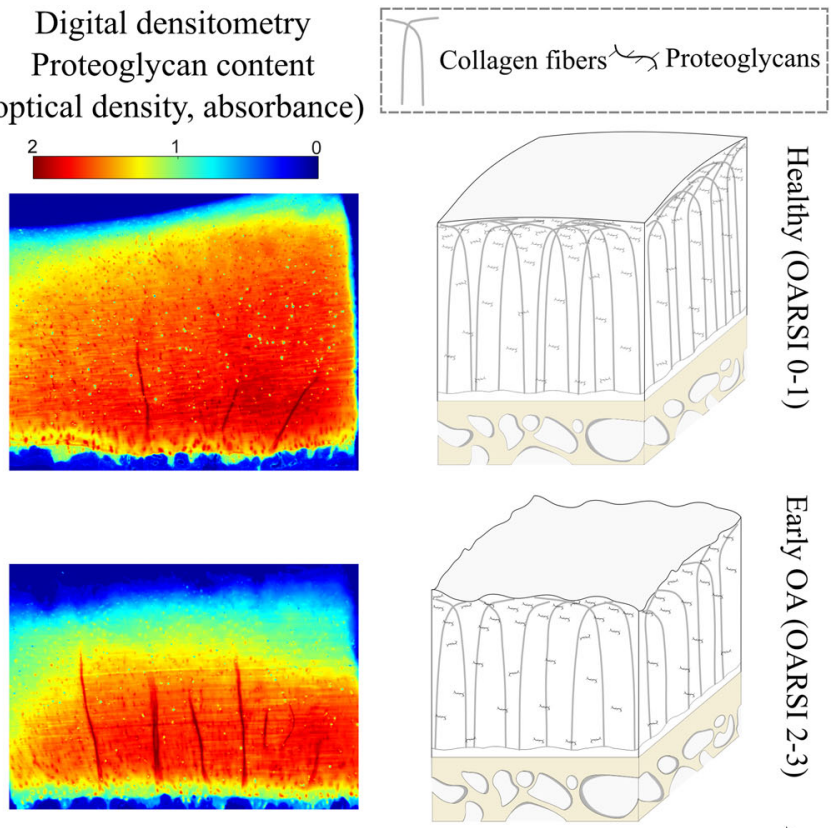

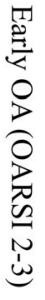

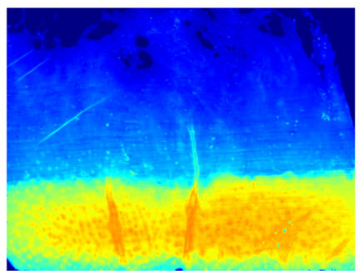

FIGURE 2. Representative images of collagen content, collagen orientation angle, and PG content in healthy (top row), early OA (middle row), and advanced OA (bottom row) cartilage.

ence at the higher frequencies $(0.05-1 \mathrm{~Hz})$. See supplementary material for the complete results.

The collagen orientation angle was negatively correlated with the equilibrium, initial instantaneous and dynamic moduli (Table 1, Supplementary Tables S1 and S2) at all tissue depths. Further, the collagen orientation angle exhibited a negative correlation with the strain-dependent instantaneous modulus at the depths of $0-20,0-50$ and $0-100 \%$ of the cartilage thickness. Furthermore, it exhibited no correlation with the phase difference at $0.005 \mathrm{~Hz}$. Interestingly, the collagen orientation angle of the superficial cartilage was positively correlated with the phase difference at higher frequencies $(0.05,0.1,0.5,0.833$ and $1 \mathrm{~Hz}$, Supplementary Table S3).

The collagen content exhibited a positive correlation with the phase difference at $0.005 \mathrm{~Hz}$ at all depths (Table 1, Supplementary Tables S1 and S2). Further, the collagen content exhibited a positive correlation with the strain-dependent instantaneous modulus only when the whole tissue was analyzed.
Structure-Composition Correlation Analysis with the Constituent-Specific Mechanical Parameters

The multivariable regression analyses for the model-derived material parameters are shown in Table 2 for the superficial $(0-10 \%)$ and bulk tissue $(0-100 \%)$. The analyses for the other depths $(0-5,0-15,0-20$ and $0-50 \%$ ) and structure-function scatter plots are presented in the supplementary material.

The PG content exhibited a positive linear correlation with the initial fibril network modulus and the non-fibrillar matrix modulus at all tissue depths (Table 2 and Supplementary Tables S1 and S2). The PG content did not exhibit any linear correlation with the initial permeability, but exhibited a negative monotonic rank correlation with the initial permeability at all tissue depths (Table 3). The PG content did not exhibit any linear or rank correlations with the strain-dependent fibril network modulus or the permeability strain-dependency coefficient. 


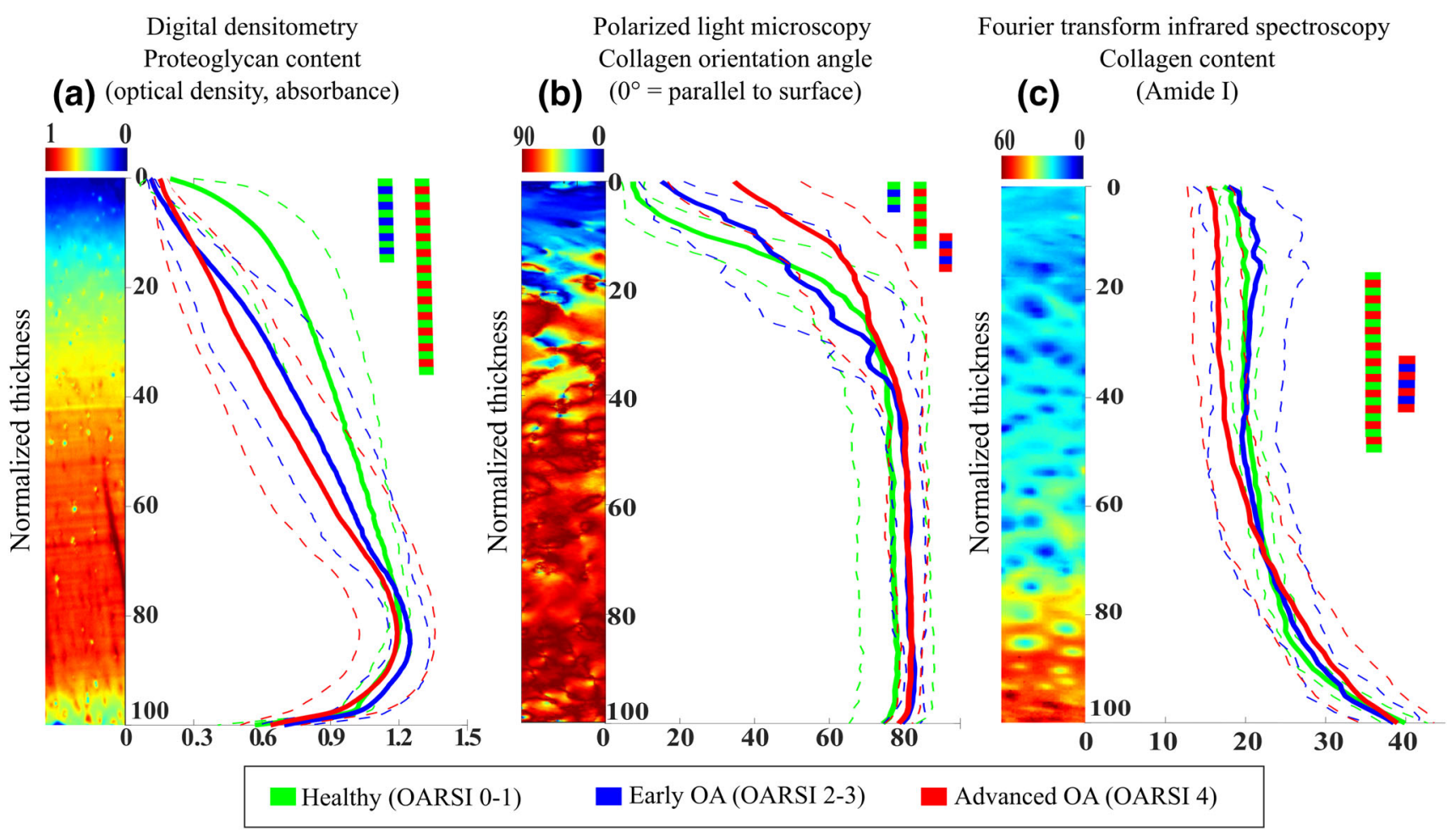

FIGURE 3. Depth-wise PG content, collagen orientation angle, and collagen content in healthy, early OA, and advanced OA cartilage. The solid line represents mean and the dotted line \pm standard deviation. Colored bars indicate depth-wise regions with statistically significant differences between the groups, $p<0.05$.

The collagen orientation angle exhibited a negative linear correlation with the initial fibril network modulus at the depths of $0-20,0-50$ and $0-100 \%$ (Table 2 and Supplementary Tables S1 and S2). The collagen orientation angle did not show a linear correlation with the initial permeability, but showed a positive rank correlation with the initial permeability at up to $15 \%$ of the tissue thickness (Table 3). Interestingly, while the collagen orientation angle did not exhibit linear or rank correlations with the strain-dependent fibril network modulus in the superficial tissue (analyzed from the surface up to $10 \%$ of the tissue thickness), there was a negative monotonic rank correlation between these parameters when deeper parts of the tissue were included in the analysis. Further, the collagen orientation angle exhibited a negative linear correlation with the permeability strain-dependency coefficient only when the bulk tissue was analyzed.

The collagen content exhibited both linear and rank correlations with the permeability strain-dependency coefficient when the analysis was conducted at the depths of $0-50$ and $0-100 \%$ (Table 2 and Supplementary Table S2).

\section{Changes in the Structure and Function of Cartilage at Different Stages of $O A$}

The PG content was smaller in the early and advanced $O A$ groups compared to the healthy group. However, the collagen disorganization (as characterized by an increase in the collagen orientation angle) occurred only in the advanced $O A$ group compared to the healthy group. Previously, we had observed that the initial fibril network modulus and the initial instantaneous modulus were lower in the early and advanced $O A$ groups compared to the healthy group (Fig. 4a). ${ }^{15}$

Furthermore, we had also previously observed that the non-fibrillar matrix modulus and equilibrium modulus were lower in the early and advanced $O A$ groups compared to the healthy group ${ }^{15}$ (Fig. 4b). In the current study, the PG content showed similar changes.

\section{DISCUSSION}

In the present study, healthy and osteoarthritic human tibial cartilage samples were characterized by investigating the depth-wise structure-function relationships. Specifically, the model-derived constituentspecific material properties and "traditional" elastic 
and viscoelastic biomechanical properties were compared with quantitative depth-wise structural and compositional properties (i.e. PG content, collagen content, and collagen orientation angle). By characterizing these, we obtained new insights on how each cartilage constituent contributes to the mechanical response of the tissue at different stages of OA. This is the first study to establish structure-function relationships in human tibial cartilage at different stages of osteoarthritis.

\section{Depth-Wise Structural and Compositional Alterations in Human Cartilage at Different Stages of OA}

We used different microscopic and spectroscopic measurements to characterize the depth-wise structure and composition (distribution of collagen fibrils and PGs). The PG content of osteoarthritic tibial cartilage was lower at the early stages of OA when compared to the healthy tissue. The loss of superficial PG content is a well-accepted sign of early OA, shown e.g. in rabbit models of $\mathrm{OA}^{5,17}$ and humans. ${ }^{49}$ Based on earlier studies and the present results, the PG loss of tibial cartilage progresses to deeper layers of cartilage at the later stages of OA.

The collagen content, on the other hand, showed no signs of changes in early OA. Consistent with our results, studies using a rabbit model of early $\mathrm{OA}^{5,33,53}$ and human cartilage ${ }^{38,49}$ showed no significant changes in the collagen content during early OA. However, some earlier rabbit OA model studies have contradictorily reported an increase in the collagen content in the deep cartilage near the bone-cartilage interface during early stages $\mathrm{OA},{ }^{17,32}$ presumably due to the upregulation of collagen synthesis. The difference to our study might be due to different species (human vs. rabbit) and different type of OA (secondary OA vs. primary OA). In the current study, the collagen content showed signs of a decrease at the late stage of OA in the middle and deep cartilage layers. Consistent with this finding, the collagen content of human patellar cartilage has been suggested to decrease only at the later stages of OA. ${ }^{49}$

PLM has been widely used to determine the orientation angle and organization of collagen fibrils., 49,49 In this study, PLM measurements indicated that collagen fibrils disorganize at the superficial cartilage $(0-$ $4 \%$ ) in early OA. Fibrillation of the cartilage surface has been reported in the literature as an early sign of $\mathrm{OA}^{4}$ In advanced OA, the disorganization of the collagen fibrils extended deeper in the tissue and was evident up to $12 \%$ of the tibial cartilage thickness. However, as we do not know the initial state of the tissue (i.e. we do not have follow-up), we cannot certainly state what mechanism causes the change in the 
TABLE 2. Structure-function relationships between the structural, compositional and FRPE material properties in the superficial $\left(0-10 \%\right.$ of the normalized tissue thickness) and bulk cartilage using multivariable linear regression $\left({ }^{\star \star} p<0.01,{ }^{\star} p<0.05\right)$.

\begin{tabular}{|c|c|c|c|c|c|c|c|c|}
\hline \multirow[b]{3}{*}{$\begin{array}{l}\text { Dependent } \\
\text { variable }\end{array}$} & \multicolumn{8}{|c|}{ Standardized regression coefficient $\beta$} \\
\hline & \multicolumn{4}{|c|}{ Superficial cartilage $(0-10 \%)$} & \multicolumn{4}{|c|}{ Bulk cartilage (0-100\%) } \\
\hline & $\begin{array}{l}\text { Adjusted } \\
\mathrm{R}^{2}\end{array}$ & $\begin{array}{l}P G \text { con- } \\
\text { tent }\end{array}$ & $\begin{array}{c}\text { Collagen orientation } \\
\text { angle }\end{array}$ & $\begin{array}{l}\text { Collagen } \\
\text { content }\end{array}$ & $\begin{array}{c}\text { Adjusted } \\
\mathrm{R}^{2}\end{array}$ & $\begin{array}{l}\text { PG con- } \\
\text { tent }\end{array}$ & $\begin{array}{c}\text { Collagen orientation } \\
\text { angle }\end{array}$ & $\begin{array}{c}\text { Collagen } \\
\text { content }\end{array}$ \\
\hline$E_{f}^{0}$ & 0.31 & $0.46^{\star *}$ & -0.32 & 0.08 & 0.30 & $0.34^{*}$ & $-0.51^{\star \star}$ & 0.32 \\
\hline$E_{f}^{\varepsilon}$ & 0.05 & -0.22 & -0.29 & 0.14 & 0.08 & 0.31 & -0.29 & 0.21 \\
\hline$E_{\mathrm{nf}}$ & 0.37 & $0.51^{* \star}$ & -0.32 & 0.05 & 0.23 & $0.46^{*}$ & -0.31 & 0.26 \\
\hline$k_{0}$ & 0.12 & -0.30 & 0.06 & 0.16 & 0.08 & -0.34 & -0.09 & 0.26 \\
\hline$M$ & 0.04 & -0.10 & -0.23 & 0.10 & 0.29 & -0.27 & $-0.37^{*}$ & $0.46^{\star \star}$ \\
\hline
\end{tabular}

These material parameters were explained and derived in Ref. 15.

$E_{f}^{0}$ initial fibril network modulus, $E_{f}^{\varepsilon}$ strain-dependent fibril network modulus, $E_{n f}$ non-fibrillar matrix modulus, $k_{0}$ initial permeability, $M$ permeability strain-dependency coefficient.

TABLE 3. Depth-wise Spearman's monotonic rank correlation analyses between the structural, compositional and FRPE material properties $\left({ }^{* *} p<0.01,{ }^{*} p<0.05\right)$.

\begin{tabular}{|c|c|c|c|c|c|c|}
\hline Parameter & Tissue depth (\%) & $E_{f}^{0}$ & $E_{f}^{\varepsilon}$ & $E_{\mathrm{nf}}$ & $k_{0}$ & $M$ \\
\hline \multirow[t]{6}{*}{ PG content } & $0-5$ & ns. & ns. & ns. & $-0.39^{\star}$ & ns. \\
\hline & $0-10$ & ns. & ns. & ns. & $-0.46^{*}$ & ns. \\
\hline & $0-15$ & ns. & ns. & ns. & $-0.45^{\star}$ & ns. \\
\hline & $0-20$ & ns. & ns. & ns. & $-0.47^{*}$ & ns. \\
\hline & $0-50$ & ns. & ns. & $0.43^{*}$ & $-0.44^{*}$ & ns. \\
\hline & $0-100$ & ns. & ns. & $0.41^{*}$ & $-0.39^{*}$ & ns. \\
\hline \multirow[t]{6}{*}{ Collagen orientation angle } & $0-5$ & ns. & ns. & $-0.54^{\star \star}$ & $0.46^{*}$ & ns. \\
\hline & $0-10$ & ns. & ns. & $-0.52^{\star \star}$ & $0.45^{\star}$ & ns. \\
\hline & $0-15$ & ns. & $-0.41^{*}$ & $-0.51^{\star *}$ & $0.39^{*}$ & ns. \\
\hline & $0-20$ & ns. & $-0.44^{*}$ & $-0.41^{*}$ & ns. & ns. \\
\hline & $0-50$ & ns. & $-0.42^{*}$ & $-0.40^{\star}$ & ns. & ns. \\
\hline & $0-100$ & ns. & $-0.39^{*}$ & ns. & ns. & ns. \\
\hline \multirow[t]{6}{*}{ Collagen content } & $0-5$ & ns. & ns. & ns. & ns. & ns. \\
\hline & $0-10$ & ns. & ns. & ns. & ns. & ns. \\
\hline & $0-15$ & ns. & ns. & ns. & ns. & ns. \\
\hline & $0-20$ & ns. & ns. & ns. & ns. & ns. \\
\hline & $0-50$ & ns. & ns. & ns. & ns. & $0.40^{*}$ \\
\hline & $0-100$ & ns. & ns. & ns. & ns. & $0.38^{*}$ \\
\hline
\end{tabular}

$E_{f}^{0}$ initial fibril network modulus, $E_{f}^{\varepsilon}$ strain-dependent fibril network modulus, $E_{n f}$ non-fibrillar matrix modulus, $k_{0}$ initial permeability, $M$ permeability strain-dependency coefficient, $n s$. not significant.

collagen orientation or what kind of initial structure may be more prone to orientation alterations when OA progresses. There might be several mechanisms behind these changes, e.g., (1) change in the PG content could lead to altered tissue loading and disruption of the normal architectural organization of the collagen network $^{27,55}$ (2) mechanical failure of collagen fibrils could lead to altered architecture and mechanical properties of the fibrillar network. ${ }^{49,54}$

\section{Associations Between the Mechanical Material Properties, Structure and Composition}

In our previous study, ${ }^{15}$ we reported that the initial fibril network modulus (i.e. pretension of the collagen fibrils in the superficial cartilage) decreased as OA progressed. In the present study, we observed that the initial fibril network modulus (and similarly the initial instantaneous modulus) correlated positively with the PG content and negatively with the collagen orientation, but it did not correlate with the collagen content. This latter observation is partly against our original hypothesis. This can be since we did not observe differences in the superficial collagen content (presumably the region related to the initial fibril network modulus) between OA and healthy groups. Thus, the correlation analysis included a too homogeneous group of samples.

The group-wise comparison revealed that the PG content was lower in the early and advanced OA groups compared to the healthy group. Simultaneously, the collagen fibril pretension was also lower in 
(a)

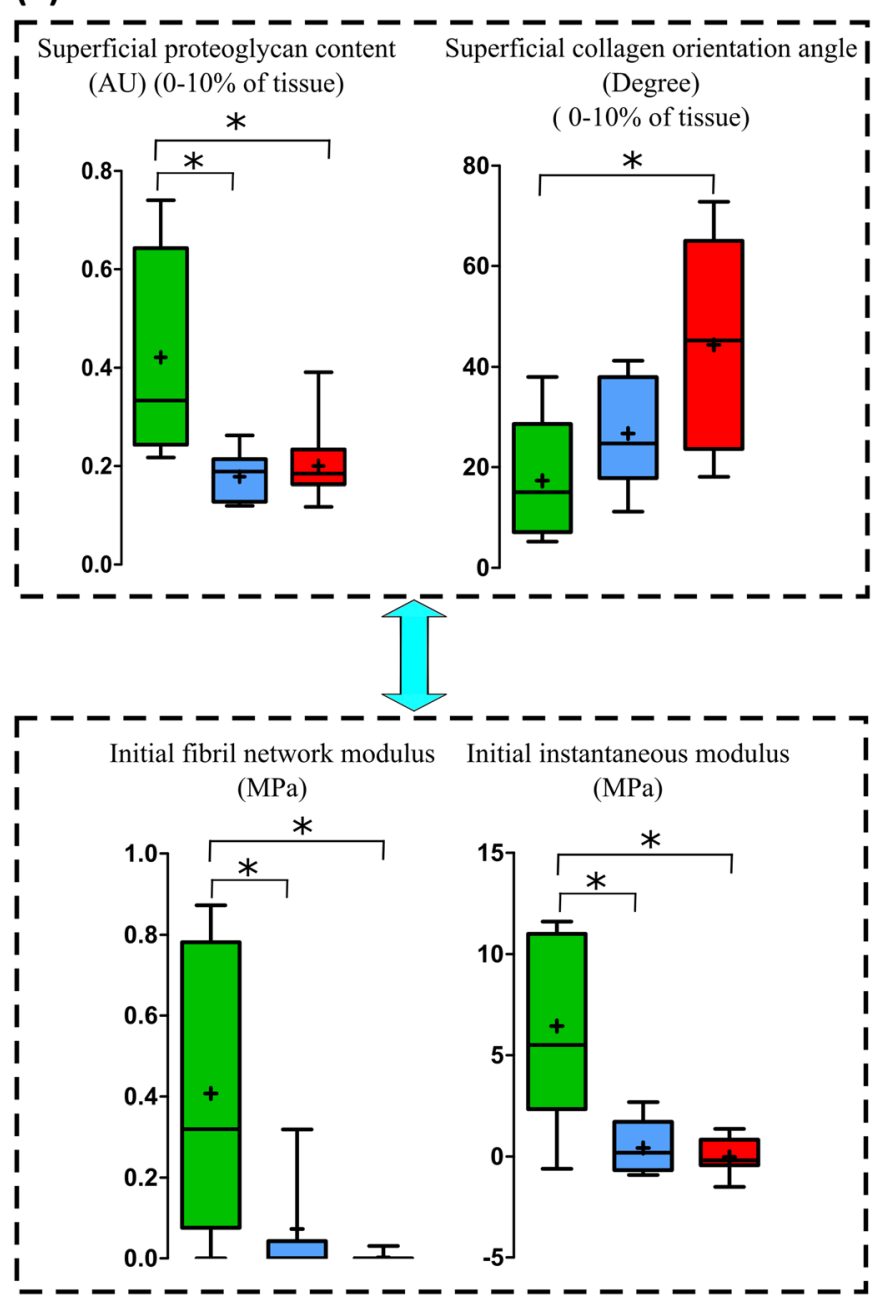

(b)

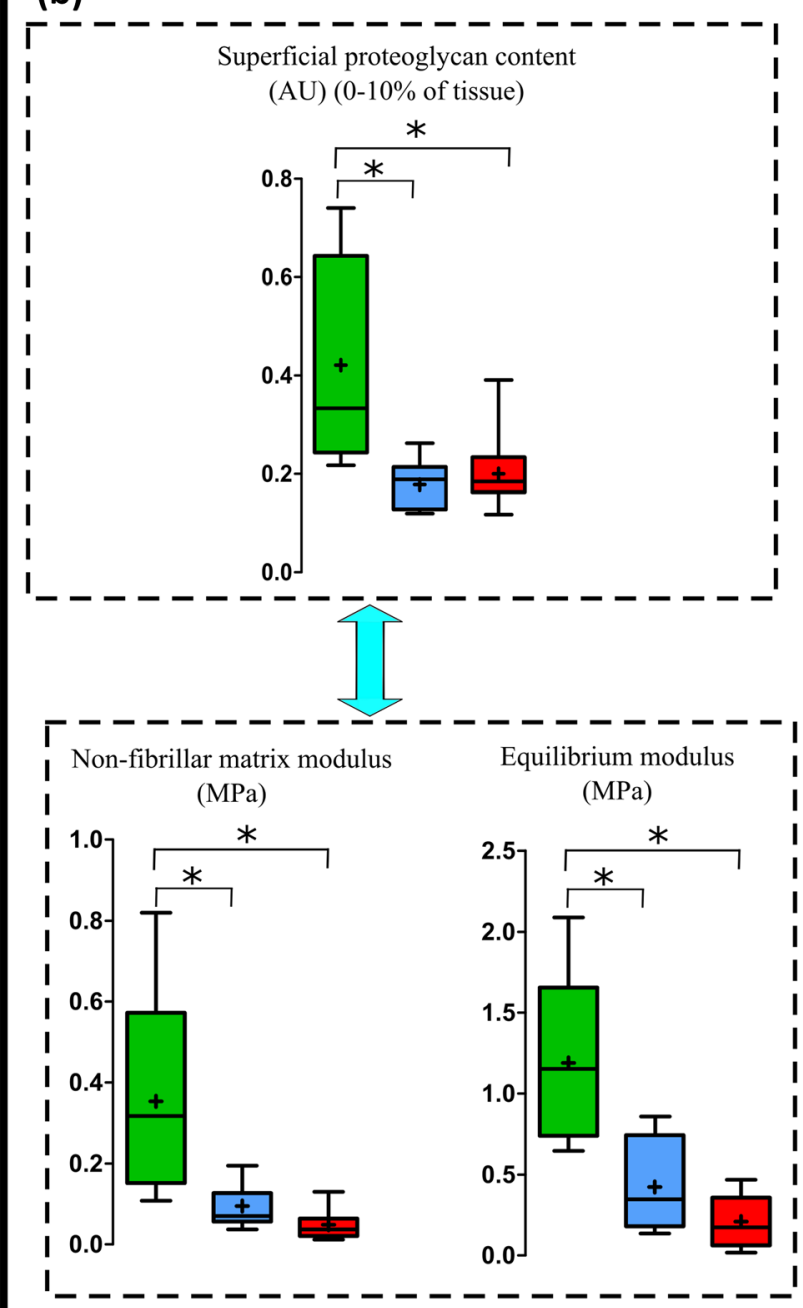

Healthy (OARSI 0-1) $\quad$ Early OA (OARSI 2-3)

Advanced OA (OARSI 4)

FIGURE 4. The summary of the observed changes in the structure and function of cartilage at different stages of OA. The results suggest that (a) the loss of collagen pretension (= the initial fibril network modulus) at different stages of OA (increasing OARSI grade) is explained by the changes in the composition (PG content) and structure (collagen orientation) of the superficial cartilage. In early $O A$ cartilage, the loss of collagen pretension is mainly regulated by the PG content, while in advanced $O A$ cartilage, it is regulated by both the PG content and collagen disorganization. The results also suggest that (b) the smaller non-fibrillar matrix and equilibrium moduli of cartilage are explained by the loss of PG content of the superficial cartilage in early and advanced $O A$ cartilage compared to healthy cartilage, ${ }^{*} p<0.05$.

the early and advanced OA groups compared to the healthy group. We hypothesize two potential mechanisms for these observations. First, when negatively charged PGs are depleted, the swelling pressure of the tissue caused by Donnan swelling will decrease. Therefore, the pretension of collagen fibrils will decrease. This is reflected by the smaller initial fibril network modulus in our model in the OA groups compared to the healthy group. Second, the collagen network is initially damaged which is observed as fibrillation of the tissue and reduction of the initial fibril network modulus. This may lead to the transportation of PGs through the loosened fibrillar network, resulting in a loss of fixed charge density and swelling pressure of the tissue. PG leakage may also be accelerated by fluid flow when cartilage is compressed. ${ }^{42}$

The collagen orientation angle in the superficial tissue increased progressively with the stage of OA, but on average at $10 \%$ of the tissue thickness, the collagen orientation was disorganized (i.e. orientation angle was greater) only in the advanced OA group compared to the healthy group. Together with earlier alterations in the initial fibril network modulus and PG content (see above and Fig. 4), this result suggests that the loss of 
the collagen pretension in the superficial tissue during early OA is mainly due to the lower tissue swelling (caused by the loss of PGs) rather than collagen disorganization. However, the loss of collagen fibril pretension in advanced OA is suggested to be regulated by both collagen disorganization and lower tissue swelling.

\section{Associations Between Viscosity (Phase Difference), Structure and Composition}

Interestingly, at a very low frequency $(0.005 \mathrm{~Hz})$, we observed (1) a negative correlation between the PG content and the phase difference (viscosity) and (2) a positive correlation between the collagen content and the phase difference. This could imply that cartilage with greater collagen content and smaller PG content could be more viscous (i.e. less elastic). The fluid pressurization and flow (i.e. poroelasticity) may also contribute less to the apparent viscoelastic mechanical response as fluid pressure is reduced at low loading frequencies $^{40}$ (see supplementary material for additional discussion and Figure S3). Thus, the contribution of collagen fibrils to apparent viscoelastic mechanical response is presumably more pronounced compared to fluid flow (poroelasticity) at low loading frequencies (e.g. $0.005 \mathrm{~Hz}$ ). This may also include the interactions between PGs and collagen fibrils and/or electrostatic repulsion between PGs themselves and these interactions can also be altered when tissue degenerates. Further, a recent study also suggested that the viscoelastic energy dissipation could be the dominant factor at lower frequencies and poroelastic energy dissipation could be the dominant factor at higher frequencies in soft connective tissues (skin and tendon) in close to micrometer scale. ${ }^{40}$ However, caution is advised when relating our results and the previous study as the length scales are substantially different $(\sim 1 \mathrm{~mm}$ vs $\sim 1 \mu \mathrm{m}$ as poroelasticity is length scale dependent i.e. we might still measure poroelastic contribution in our measurements) and different tissues were characterized.

At higher frequencies (ranging from 0.05 to $1 \mathrm{~Hz}$ ), the collagen content did not correlate with the phase difference. Instead, we observed (1) a positive correlation between the collagen orientation angle and the phase difference and (2) a negative correlation between the PG content and the phase difference. The first observation would suggest that cartilage with a less organized structure could dissipate more energy (as it is more viscous) at higher frequencies. Further, as in the very low loading frequency case, our data suggest that at high frequencies the smaller PG content also increases viscous mechanical behavior. However, the viscous behavior at high loading frequencies is most likely controlled by the poroelasticity. We also observed that greater collagen disorganization and smaller PG content were associated with an increase in cartilage permeability at the superficial cartilage. This suggests that the loss of the PGs and the disorganization of collagen fibrils facilitate the fluid flow in cartilage. This finding was partly against our original hypothesis in which we stated that viscous properties of cartilage are controlled only by the PG content. Finally, we also observed that the phase difference and dynamic modulus reached a plateau at around $0.1 \mathrm{~Hz}$, suggesting that the dynamic properties would be similar at frequencies higher than $1 \mathrm{~Hz}$.

Based on our observations and the related conclusions it seems that fluid flow-independent collagen viscoelasticity may be more dominant on the apparent viscous response of the tissue at low frequencies while the fluid flow-dependent viscoelasticity (i.e. poroelasticity) is dominant on the apparent viscous response at higher frequencies. However, fluid flow-dependent viscoelasticity may still contribute to the viscous response at lower frequencies to some extent.

The positive rank correlation between the permeability strain-dependency coefficient and the collagen content suggests that higher content (as collagen is presumably more densely packed) impair the fluid flow at high compressive strains, thus reducing permeability as a function of strain. This can be the case especially for healthy samples, which had higher collagen content at middle and deep layers compared to advanced $O A$ samples. This finding is also consistent with a recent human hip joint cartilage study ${ }^{31}$ which reported a similar positive correlation between the permeability strain-dependency coefficient and collagen content.

\section{Limitations and Applications}

Generally, cartilage becomes thinner in the advanced stages of OA. ${ }^{16}$ On the other hand, for early OA animal models, investigators have also reported an increase in tissue thickness due to the disorganization of the collagen network and cartilage swelling. ${ }^{7,11,43}$ This thickness difference could potentially affect depth-wise analysis when comparing structural and compositional properties between OA groups as a function of the cartilage thickness. However, in our study, the sample thicknesses were not statistically different between the groups ${ }^{15}(p$ value $=0.99$ for linear mixed model). A recent study also showed that the thickness of human tibial cartilage was not associated with the severity of cartilage degeneration (OARSI grades between 0 and 4 ). ${ }^{13}$ Cartilage thinning is associated with very late stages of OA, e.g. OARSI 5-6. ${ }^{44}$ Moreover, biomechanical testing and analysis of tissue composition and structure were consistently 
performed to the normalized tissue thickness. Therefore, the comparison between the structural, compositional and functional properties in this study should be plausible.

To thoroughly characterize the structure-function relations during the progression of human $\mathrm{OA}$, the initial condition of cartilage must be known and the OA progression of that sample must be followed. This could be possible by in vivo imaging, such as MRI, but then a detailed and high-resolution characterization of tissue structure and function, as done here, would not be possible.

Another limitation of the current study is related to the number of samples in different OA groups. For instance, there were 17 samples in the advanced $O A$ group against only 5 samples in the healthy group. This was since the samples were obtained from relatively old cadavers (age $71.4 \pm 5.2$ years). Due to this our sample pool might be too homogenous which is partly seen in our concentrated data points in the scatter plots (see supplementary material Figure S2). To elucidate the strength of our statistical conclusions, we conducted an additional power analysis for the data. We observed that the linear mixed-effects model had a very high power (ranging from 0.94 to 0.99 , Supplementary Material Table S4). In addition, the observed power for identifying the group-wise differences was also relatively high (ranging from 0.47 to 0.97 ) for the significantly different groups (Supplementary Material Table S5).

Recent studies have developed sophisticated knee joint models for the prediction of OA progression. ${ }^{18,34}$ However, none of the earlier models have included realistic human cartilage properties. The knowledge from the current paper can improve the accuracy of those models. In addition, this knowledge is important for multiscale studies investigating cell-tissue interactions at different tissue depths. ${ }^{8}$

Further, a better understanding of the associations between the mechanical properties and biochemical content and structure within native human cartilage may help to develop methods for non-destructive evaluation of cartilage. ${ }^{20}$ In the future, we aim to apply computational methods to estimate the function of cartilage based on the composition and structure, thus, the direct measurement of tissue function (e.g. indentation) would not be needed.

\section{Conclusion}

In conclusion, the present study provides novel information about the complex structure-function relationships in human tibial cartilage. The results suggest that the loss of collagen fibrils pretension in the superficial cartilage is controlled by the loss of PG content (i.e. swelling pressure) at early $\mathrm{OA}$ and by the combination of the loss of PG content and greater disorganization of collagen fibrils at advanced/late OA. Furthermore, the results suggest that different mechanisms (fluid flow-independent collagen viscoelasticity and poroelasticity) may contribute to cartilage viscosity in low and high frequencies.

\section{ELECTRONIC SUPPLEMENTARY MATERIAL}

The online version of this article (https://doi.org/10. 1007/s10439-020-02559-0) contains supplementary material, which is available to authorized users.

\section{ACKNOWLEDGMENTS}

Open access funding provided by University of Eastern Finland (UEF) including Kuopio University Hospital. Santtu Mikkonen, Ph.D., and Noel Barengo, $\mathrm{Ph} . \mathrm{D}$., are acknowledged for statistical consultation. This work was supported by Academy of Finland (Grants 286526, 324529 and 268378); Sigrid Juselius Foundation; University of Oulu (strategic funding); Finnish Cultural Foundation, North Savo Regional Fund (Grant 65191841) and Central Fund (Grant 191044); Maire Lisko Foundation; Otto A. Malm Foundation; Emil Aaltonen Foundation (Grant 200016); and Alfred Kordelin Foundation (Grant 190111).

\section{CONFLICT OF INTEREST}

The authors declare no conflicts of interest.

\section{OPEN ACCESS}

This article is licensed under a Creative Commons Attribution 4.0 International License, which permits use, sharing, adaptation, distribution and reproduction in any medium or format, as long as you give appropriate credit to the original author(s) and the source, provide a link to the Creative Commons licence, and indicate if changes were made. The images or other third party material in this article are included in the article's Creative Commons licence, unless indicated otherwise in a credit line to the material. If material is not included in the article's Creative Commons licence and your intended use is not permitted by statutory regulation or exceeds the permitted use, you will need to obtain permission directly from the copyright holder. To view a copy of this licence, visit http://crea tivecommons.org/licenses/by/4.0/. 


\section{REFERENCES}

${ }^{1}$ Appleyard, R. C., P. Ghosh, and M. V. Swain. Biomechanical, histological and immunohistological studies of patellar cartilage in an ovine model of osteoarthritis induced by lateral meniscectomy. Osteoarthr. Cartil. 7:281294, 1999.

${ }^{2}$ Armstrong, S., R. Read, and P. Ghosh. The effects of intraarticular hyaluronan on cartilage and subchondral bone changes in an ovine model of early osteoarthritis. $J$. Rheumatol. 21:680-688, 1994.

${ }^{3}$ Athanasiou, K. A., M. P. Rosenwasser, J. A. Buckwalter, T. I. Malinin, and V. C. Mow. Interspecies comparisons of in situ intrinsic mechanical properties of distal femoral cartilage. J. Orthop. Res. 9:330-340, 1991.

${ }^{4}$ Bi, X., G. Li, S. B. Doty, and N. P. Camacho. A novel method for determination of collagen orientation in cartilage by Fourier transform infrared imaging spectroscopy (FT-IRIS). Osteoarthr. Cartil. 13:1050-1058, 2005.

${ }^{5}$ Bi, X., X. Yang, M. P. G. Bostrom, D. Bartusik, S. Ramaswamy, K. W. Fishbein, R. G. Spencer, and N. P. Camacho. Fourier transform infrared imaging and MR microscopy studies detect compositional and structural changes in cartilage in a rabbit model of osteoarthritis. Anal. Bioanal. Chem. 387:1601-1612, 2007.

${ }^{6}$ Brown, W. E., G. D. DuRaine, J. C. Hu, and K. A. Athanasiou. Structure-function relationships of fetal ovine articular cartilage. Acta Biomater. 87:235-244, 2019.

${ }^{7}$ Calvo, E., I. Palacios, E. Delgado, J. Ruiz-Cabello, P. Hernandez, O. Sanchez-Pernaute, J. Egido, and G. Herrero-Beaumont. High-resolution MRI detects cartilage swelling at the early stages of experimental osteoarthritis. Osteoarthr. Cartil. 9:463-472, 2001.

${ }^{8}$ Chen, J., J. Irianto, S. Inamdar, P. Pravincumar, D. A. Lee, D. L. Bader, and M. M. Knight. Cell mechanics, structure, and function are regulated by the stiffness of the three-dimensional microenvironment. Biophys. $J$. 103:1188-1197, 2012

${ }^{9}$ Chiu, L. L. Y., R. Giardini-Rosa, J. F. Weber, S. L. Cushing, and S. D. Waldman. Comparisons of auricular cartilage tissues from different species. Ann. Otol. Rhinol. Laryngol. 126:819-828, 2017.

${ }^{10}$ Collins, K. H., R. A. Reimer, R. A. Seerattan, T. R. Leonard, and W. Herzog. Using diet-induced obesity to understand a metabolic subtype of osteoarthritis in rats. Osteoarthr. Cartil. 23:957-965, 2015.

${ }^{11}$ Cotofana, S., R. Buck, W. Wirth, F. Roemer, J. Duryea, M. Nevitt, and F. Eckstein. Cartilage thickening in early radiographic knee osteoarthritis: a within-person, betweenknee comparison. Arthritis Care Res. 64:1681-1690, 2012.

${ }^{12}$ Danso, E. K., J. M. T. Oinas, S. Saarakkala, S. Mikkonen, J. Töyräs, and R. K. Korhonen. Structure-function relationships of human meniscus. J. Mech. Behav. Biomed. Mater. 67:51-60, 2017.

${ }^{13}$ Deng, B., F. Wang, L. Yin, C. Chen, L. Guo, H. Chen, X. Gong, Y. Li, and L. Yang. Quantitative study on morphology of calcified cartilage zone in OARSI 0 4 cartilage from osteoarthritic knees. Curr. Res. Transl. Med. 64:149154, 2016.

${ }^{14}$ Desrochers, J., M. A. Amrein, and J. R. Matyas. Structural and functional changes of the articular surface in a post- traumatic model of early osteoarthritis measured by atomic force microscopy. J. Biomech. 43:3091-3098, 2010.

${ }^{15}$ Ebrahimi, M., S. Ojanen, A. Mohammadi, M. A. Finnilä, A. Joukainen, H. Kröger, S. Saarakkala, R. K. Korhonen, and P. Tanska. Elastic, viscoelastic and fibril-reinforced poroelastic material properties of healthy and osteoarthritic human tibial cartilage. Ann. Biomed. Eng. 47:953966, 2019.

${ }^{16}$ Eckstein, F., W. Wirth, and M. C. Nevitt. Recent advances in osteoarthritis imaging: the osteoarthritis initiative. Nat. Rev. Rheumatol. 8:622-630, 2012.

${ }^{17}$ Florea, C., M. K. H. Malo, J. Rautiainen, J. T. A. Mäkelä, J. M. Fick, M. T. Nieminen, J. S. Jurvelin, A. Davidescu, and R. K. Korhonen. Alterations in subchondral bone plate, trabecular bone and articular cartilage properties of rabbit femoral condyles at 4 weeks after anterior cruciate ligament transection. Osteoarthr. Cartil. 23:414-422, 2015.

${ }^{18}$ Halonen, K. S., M. E. Mononen, J. S. Jurvelin, J. Töyräs, and R. K. Korhonen. Importance of depth-wise distribution of collagen and proteoglycans in articular cartilage: a 3D finite element study of stresses and strains in human knee joint. J. Biomech. 46:1184-1192, 2013.

${ }^{19}$ Hänninen, N., J. Rautiainen, L. Rieppo, S. Saarakkala, and M. J. Nissi. Orientation anisotropy of quantitative MRI relaxation parameters in ordered tissue. Sci. Rep. 7:9606, 2017.

${ }^{20}$ Haudenschild, A. K., B. E. Sherlock, X. Zhou, J. C. Hu, J. K. Leach, L. Marcu, and K. A. Athanasiou. Nondestructive fluorescence lifetime imaging and time-resolved fluorescence spectroscopy detect cartilage matrix depletion and correlate with mechanical properties. Eur. Cells Mater. 36:30-43, 2018.

${ }^{21}$ Heard, B. J., K. I. Barton, M. Chung, Y. Achari, N. G. Shrive, C. B. Frank, and D. A. Hart. Single intra-articular dexamethasone injection immediately post-surgery in a rabbit model mitigates early inflammatory responses and post-traumatic osteoarthritis-like alterations. J. Orthop. Res. 33:1826-1834, 2015.

${ }^{22}$ Hosseini, S. M., W. Wilson, K. Ito, and C. C. Van Donkelaar. A numerical model to study mechanically induced initiation and progression of damage in articular cartilage. Osteoarthr. Cartil. 22:95-103, 2014.

${ }^{23}$ Julkunen, P., R. K. Korhonen, M. J. Nissi, and J. S. Jurvelin. Mechanical characterization of articular cartilage by combining magnetic resonance imaging and finite-element analysis: a potential functional imaging technique. Phys. Med. Biol. 53:2425, 2008.

${ }^{24}$ Julkunen, P., W. Wilson, H. Isaksson, J. S. Jurvelin, W. Herzog, and R. K. Korhonen. A review of the combination of experimental measurements and fibril-reinforced modeling for investigation of articular cartilage and chondrocyte response to loading. Comput. Math. Methods Med. 2013.

${ }^{25}$ Korhonen, R. K., M. S. Laasanen, J. Töyräs, R. Lappalainen, H. J. Helminen, and J. S. Jurvelin. Fibril reinforced poroelastic model predicts specifically mechanical behavior of normal, proteoglycan depleted and collagen degraded articular cartilage. J. Biomech. 36:1373-1379, 2003.

${ }^{26}$ Lawrence, R. C., D. T. Felson, C. G. Helmick, L. M. Arnold, H. Choi, R. A. Deyo, S. Gabriel, R. Hirsch, M. C. Hochberg, and G. G. Hunder. Estimates of the prevalence 
of arthritis and other rheumatic conditions in the United States: part II. Arthritis Rheum 58:26-35, 2008.

${ }^{27} \mathrm{Li}$, L., M. D. Buschmann, and A. Shirazi-Adl. The role of fibril reinforcement in the mechanical behavior of cartilage. Biorheology 39:89-96, 2002.

${ }^{28} \mathrm{Li}, \mathrm{L}$. P., and W. Herzog. The role of viscoelasticity of collagen fibers in articular cartilage: theory and numerical formulation. Biorheology 41:181-194, 2004.

${ }^{29}$ Lu, X. L., and V. C. Mow. Biomechanics of articular cartilage and determination of material properties. Med. Sci. Sport. Exerc. 40:193-199, 2008.

${ }^{30} \mathrm{Mak}, \mathrm{A}$. F. The apparent viscoelastic behavior of articular cartilage - the contributions from the intrinsic matrix viscoelasticity and interstitial fluid flows. J. Biomech. Eng. 108:123-130, 1986

${ }^{31}$ Mäkelä, J. T. A., M. R. J. Huttu, and R. K. Korhonen. Structure-function relationships in osteoarthritic human hip joint articular cartilage. Osteoarthr. Cartil. 20:1268$1277,2012$.

${ }^{32}$ Mäkelä, J. T. A., Z. S. Rezaeian, S. Mikkonen, R. Madden, S.-K. Han, J. S. Jurvelin, W. Herzog, and R. K. Korhonen. Site-dependent changes in structure and function of lapine articular cartilage 4 weeks after anterior cruciate ligament transection. Osteoarthr. Cartil. 22:869-878, 2014.

${ }^{33}$ Mäkitalo, J., S. Saarakkala, L. Rieppo, S. K. Han, W. Herzog, and R. K. Korhonen. Can collagen fibrillation or proteoglycan depletion of cartilage explain changed deformation behavior of chondrocytes 9 weeks after anterior cruciate ligament transaction? Trans. Orthop. Res. Soc. 36:2026, 2011

${ }^{34}$ Mononen, M. E., P. Tanska, H. Isaksson, and R. K. Korhonen. New algorithm for simulation of proteoglycan loss and collagen degeneration in the knee joint: data from the osteoarthritis initiative. J. Orthop. Res. 36:1673-1683, 2018.

${ }^{35}$ Mow, V. C., M. H. Holmes, and W. M. Lai. Fluid transport and mechanical properties of articular cartilage: a review. J. Biomech. 17:377-394, 1984.

${ }^{36}$ Mow, V. C., and R. Huiskes. Basic Orthopaedic Biomechanics \& Mechano-Biology. Philadelphia: Lippincott Williams \& Wilkins, 2005.

${ }^{37}$ Mow, V. C., A. Ratcliffe, and A. R. Poole. Cartilage and diarthrodial joints as paradigms for hierarchical materials and structures. Biomaterials 13:67-97, 1992.

${ }^{38}$ Nelson, F., R. C. Billinghurst, I. Pidoux, A. Reiner, M. Langworthy, M. McDermott, T. Malogne, D. F. Sitler, N. R. Kilambi, and E. Lenczner. Early post-traumatic osteoarthritis-like changes in human articular cartilage following rupture of the anterior cruciate ligament. Osteoarthr. Cartil. 14:114-119, 2006.

${ }^{39}$ Nieminen, M. T., J. Rieppo, J. Töyräs, J. M. Hakumäki, J. Silvennoinen, M. M. Hyttinen, H. J. Helminen, and J. S. Jurvelin. T2 relaxation reveals spatial collagen architecture in articular cartilage: a comparative quantitative MRI and polarized light microscopic study. Magn. Reson. Med. 46:487-493, 2001.

${ }^{40}$ Oftadeh, R., B. K. Connizzo, H. T. Nia, C. Ortiz, and A. J. Grodzinsky. Biological connective tissues exhibit viscoelastic and poroelastic behavior at different frequency regimes: application to tendon and skin biophysics. Acta Biomater. 70:249-259, 2018.

${ }^{41}$ Ojanen, S. P., M. A. J. Finnilä, A. E. Reunamo, A. P. Ronkainen, S. Mikkonen, W. Herzog, S. Saarakkala, and
R. K. Korhonen. Site-specific glycosaminoglycan content is better maintained in the pericellular matrix than the extracellular matrix in early post-traumatic osteoarthritis. PLoS ONE 13:e0196203, 2018.

${ }^{42}$ Orozco, G. A., P. Tanska, C. Florea, A. J. Grodzinsky, and R. K. Korhonen. A novel mechanobiological model can predict how physiologically relevant dynamic loading causes proteoglycan loss in mechanically injured articular cartilage. Sci. Rep. 8:1-16, 2018.

${ }^{43}$ Panula, H. E., M. M. Hyttinen, J. P. A. Arokoski, T. K. Långsjö, A. Pelttari, I. Kiviranta, and H. J. Helminen. Articular cartilage superficial zone collagen birefringence reduced and cartilage thickness increased before surface fibrillation in experimental osteoarthritis. Ann. Rheum. Dis. 57:237-245, 1998

${ }^{44}$ Pritzker, K. P. H., S. Gay, S. A. Jimenez, K. Ostergaard, J.P. Pelletier, P. A. Revell, D. Salter, and W. B. Van den Berg. Osteoarthritis cartilage histopathology: grading and staging. Osteoarthr. Cartil. 14:13-29, 2006.

${ }^{45}$ Quiroga, J. M. P., W. Wilson, K. Ito, and C. C. van Donkelaar. Relative contribution of articular cartilage's constitutive components to load support depending on strain rate. Biomech. Model. Mechanobiol. 16:151-158, 2017.

${ }^{46}$ Rieppo, J., J. Hallikainen, J. S. Jurvelin, I. Kiviranta, H. J. Helminen, and M. M. Hyttinen. Practical considerations in the use of polarized light microscopy in the analysis of the collagen network in articular cartilage. Microsc. Res. Tech. 71:279-287, 2008.

${ }^{47}$ Rieppo, J., J. Töyräs, M. T. Nieminen, V. Kovanen, M. M. Hyttinen, R. K. Korhonen, J. S. Jurvelin, and H. J. Helminen. Structure-function relationships in enzymatically modified articular cartilage. Cells Tissues Organs 175:121-132, 2003.

${ }^{48}$ Saarakkala, S., and P. Julkunen. Specificity of Fourier transform infrared (FTIR) microspectroscopy to estimate depth-wise proteoglycan content in normal and osteoarthritic human articular cartilage. Cartilage 1:262-269, 2010.

${ }^{49}$ Saarakkala, S., P. Julkunen, P. Kiviranta, J. Mäkitalo, J. S. Jurvelin, and R. K. Korhonen. Depth-wise progression of osteoarthritis in human articular cartilage: investigation of composition, structure and biomechanics. Osteoarthr. Cartil. 18:73-81, 2010.

${ }^{50}$ Sarin, J. K., N. te Moller, H. Brommer, R. van Weeren, I. Mancini, J. Malda, I. O. Afara, and J. Töyräs. Spectroscopic evaluation of post-traumatic osteoarthritis in Shetland ponies. Optics InfoBase Conference Paper. Part F90O:4-5, 2018

${ }^{51}$ Soltz, M. A., and G. A. Ateshian. Interstitial fluid pressurization during confined compression cyclical loading of articular cartilage. Ann. Biomed. Eng. 28:150-159, 2000.

${ }^{52}$ Stewart, R. C., J. T. J. Honkanen, H. T. Kokkonen, V. Tiitu, S. Saarakkala, A. Joukainen, B. D. Snyder, J. S. Jurvelin, M. W. Grinstaff, and J. Töyräs. contrast-enhanced computed tomography enables quantitative evaluation of tissue properties at intrajoint regions in cadaveric knee cartilage. Cartilage 8:391-399, 2017.

${ }^{53}$ Turunen, S. M., S.-K. Han, W. Herzog, and R. K. Korhonen. Cell deformation behavior in mechanically loaded rabbit articular cartilage 4 weeks after anterior cruciate ligament transection. Osteoarthr. Cartil. 21:505513,2013 
${ }^{54}$ Turunen, S. M., M. J. Lammi, S. Saarakkala, S.-K. Han, W. Herzog, P. Tanska, and R. K. Korhonen. The effect of collagen degradation on chondrocyte volume and morphology in bovine articular cartilage following a hypotonic challenge. Biomech. Model. Mechanobiol. 12:417-429, 2013.

${ }^{55}$ Urban, J. P. G., A. Maroudas, M. T. Bayliss, and J. Dillon. Swelling pressures of proteoglycans at the concentrations found in cartilaginous tissues. Biorheology 16:447-464, 1979.

Publisher's Note Springer Nature remains neutral with regard to jurisdictional claims in published maps and institutional affiliations. 